DOI 10.4171/JEMS/189

Hitoshi Nakada

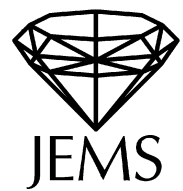

\title{
On the Lenstra constant associated to the Rosen continued fractions
}

Received July 17, 2007 and in revised form April 17, 2008

Abstract. The purpose of this paper is to describe the relation between the Legendre and the Lenstra constants. Indeed we show that they are equal whenever the Legendre constant exists; in particular, this holds for both Rosen continued fractions and $\alpha$-continued fractions. We also give the explicit value of the entropy of the Rosen map with respect to the absolutely continuous invariant probability measure.

Keywords. Continued fractions, Hecke group, geodesic flows

\section{Introduction}

Let $x$ be an irrational number in $[0,1]$. We denote by $p_{n} / q_{n}$ the $n$-th principal regular continued fraction convergent of $x$ and recall the following.

Theorem (Legendre). Suppose $p$ and $q(>0)$ are relatively prime integers and $|x-p / q|$ $<1 / 2 q^{2}$. Then $p / q$ is a principal convergent to $x$. On the other hand, for any $c>1 / 2$, there exist $x$ and $p / q$, which is not a principal convergent, such that $|x-p / q|<c / q^{2}$.

In this situation, we say that $1 / 2$ is the Legendre constant of regular continued fractions. Now we consider the error of the principal convergents. We put

$$
\Theta_{n}=q_{n}^{2}\left|x-\frac{p_{n}}{q_{n}}\right| .
$$

The following fact was proved in [2].

Theorem (Bosma, Jager and Wiedijk, 1983). For a.e. $x$, the limit

$$
\lim _{n \rightarrow \infty} \frac{1}{n} \sum_{j=1}^{\infty}\left\{1 \leq j \leq n: \Theta_{j} \leq t\right\}
$$

H. Nakada: Department of Mathematics, Keio University, Hiyoshi, Kohoku-ku, Yokohama 223-8522, Japan; e-mail: nakada@math.keio.ac.jp

Mathematics Subject Classification (2010): 11K50, 37A45 
exists for any $t, 0 \leq t \leq 1$, and is equal to

$$
F(t)= \begin{cases}\frac{t}{\ln 2}, & 0 \leq t \leq 1 / 2 \\ \frac{1}{\ln 2}(1-t+\ln 2 t), & 1 / 2 \leq t \leq 1 .\end{cases}
$$

We note that $F(t)$ is linear in $0 \leq t \leq 1 / 2$ and not in $1 / 2 \leq t \leq 1$. We say that $1 / 2$ is the Lenstra constant of regular continued fractions because this fact was conjectured by Doeblin in 1940 [4] implicitly, and then proved by H. W. Lenstra in 1981; see [7, p. 252]. Legendre and Lenstra constants can be defined for other types of continued fraction expansions in a similar manner (e.g. for $\alpha$-expansions, [16], [2]). In general, it is not hard to show that the Lenstra constant exists and is at least as large as the Legendre constant for each type of continued fraction expansion whenever the Legendre constant exists. However, a number of examples indicate that these constants may be equal; for example see [8], [9]. In this paper, we show that indeed the Lenstra constant is equal to the Legendre constant. Let us explain why their equality is important. The classical Hurwitz theorem states that for every irrational number $x$ there are infinitely many rationals $p / q$ for which

$$
\left|x-\frac{p}{q}\right|<\frac{1}{\sqrt{5}} \frac{1}{q^{2}}
$$

and that the constant $1 / \sqrt{5}$ is the maximum value for which this property holds. On the other hand, a result of Borel states that for every $x$ and every $n \geq 1$ one has $\min \left\{\Theta_{n-1}, \Theta_{n}, \Theta_{n+1}\right\}<1 / \sqrt{5}$. Now Hurwitz' result is an immediate consequence of the result by Borel and Legendre stated above. Here we should note that $1 / \sqrt{5}<1 / 2$. In fact, Legendre's result implies that if one wants to approximate irrational numbers $x$ by rationals $p / q$, one is-due to Legendre's result- 'forced' to use continued fraction convergents. Furthermore, Borel's result (and also the Lenstra conjecture) shows that there are in fact many good rational approximations, which can be easily obtained via the Gauss map which induces the continued fraction expansions for irrational numbers $0<x<1$. Since the Lenstra constant can be calculated in a straightforward way, due to the result in this paper, one knows the Legendre constant quite easily.

Motivation of this paper is the metrical theory of the Rosen continued fractions associated to Hecke groups. It is possible to define the Legendre constants in this case even though the convergents of the Rosen continued fractions are not rational numbers. Indeed, in 1985 J. Lehner [13] claimed that the Legendre constant of Rosen continued fractions is greater than or equal to $1 / 2$ for Hecke groups of any indices. However, the proof was not correct (see [14], actually the correct value is less than 1/2) and a lower estimate was given in [21] (where the constants depend on the indices of Hecke groups). On the other hand, the Lenstra constant was given in [3] (and [17] for the even indices case). Also [17] claimed (without proof) that the Lenstra constant and the Legendre constant are the same for each Hecke group of even index. Here we note that Corollary 4.1 of [3] did not say that the constant is the best possible one (which means it is the Lenstra constant), but it is not hard to see that this is indeed the case. We refer to [10] for this point. 
In the following, we show that the Lenstra constant is equal to the Legendre constant for the Rosen continued fractions. In the next section, we introduce a generalized Diophantine approximation problem associated to a zonal Fuchsian group and give a law of large numbers for solutions of a Diophantine inequality. This ensures the existence of the Lenstra constant whenever the Legendre constant exists, and also implies that the Lenstra constant is at least as large as the Legendre constant. In $\S 3$, we prove the equality of these two constants, by showing that the Lenstra constant cannot be larger than the Legendre constant. As a corollary (of the proof), we get the explicit value of the entropy of the Rosen map. Finally, we note that the same result holds for $\alpha$-continued fractions, $0<\alpha \leq 1 / 2$. For $1 / 2 \leq \alpha \leq 1$, this result was shown by C. Kraaikamp [9] in a different way. Recently, R. Natsui [19] showed the existence of the Legendre constant for any $0<\alpha \leq 1 / 2$ and thus we can apply the method of this paper to show the equality of these two constants. We also note that S. Ito [8] determined the Legendre constant of mediant convergents. We stress the difference between the concepts of the two constants. The Legendre constant is determined by a property which holds for all $x$, without exceptional point; on the other hand, the Lenstra constant comes from a metrical property which only holds for almost all points.

Finally, we note the relation between the Legendre constant and the Hurwitz constant. In [11], the authors derive a Borel type result for the Rosen continued fractions. Since the Legendre constant for the Rosen fractions is smaller than the Borel constant found by Kraaikamp et al., the result of these authors does not imply the Hurwitz-type result for the Rosen fractions, which was previously obtained by Haas and Series. In [10], the authors look at a different map associated with the Rosen fractions, and for this map the Legendre constant is larger than the Borel constant, and-as in the case of the regular continued fraction expansion-the result by Haas and Series follows.

\section{Generalized Diophantine approximation}

Let $\Gamma$ be a finitely generated Fuchsian group acting on the complex upper half-plane $\mathbb{H}^{2}$, $L$ the set of limit points of $\Gamma$, and $P$ the set of parabolic points. We assume that $\infty \in P$.

An element $g \in \Gamma$ can be viewed as a $2 \times 2$ real matrix

$$
\left(\begin{array}{ll}
a & b \\
c & d
\end{array}\right)
$$

of determinant 1. We write $a=a(g), b=b(g), c=c(g)$ and $d=d(g)$.

J. Lehner [12] proved that there exists a positive number $t$ depending on $\Gamma$ such that

$$
\sharp\left\{g(\infty):|x-g(\infty)|<t / c^{2}(g), g \in \Gamma\right\}=\infty
$$

for any $x \in L \backslash P$. He also proved that if $\Gamma$ is of the first kind $(L=\mathbb{R})$, then for any sequence $\left\{\varepsilon_{n}\right\}$ of positive numbers and a.e. $x \in L \backslash P$, there exists a sequence $\left\{g_{n}\right\}$ in $\Gamma$ such that

$$
\left|x-g_{n}(\infty)\right|<\varepsilon_{n} / c^{2}\left(g_{n}\right)
$$


Moreover, S. J. Patterson [20] proved a kind of Khinchin's theorem when $\Gamma$ is of the first kind: for example, his result implies that

$$
\sharp\left\{g(\infty):|x-g(\infty)|<(\ln |c(g)|) / c^{2}(g), g \in \Gamma\right\}=\infty
$$

for a.e. $x \in \mathbb{R} \backslash P$.

We shall estimate the asymptotic number of solutions $g(\infty)$ of

$$
|x-g(\infty)|<t / c^{2}(g), \quad g \in \Gamma,
$$

for some positive real number $t$ and a.e. $x \in \mathbb{R} \backslash P$. To do this, we consider a relation among the Diophantine inequality, geodesics of $\mathbb{H}^{2}$, and geodesics of $\mathbb{H}^{2} / \Gamma$. We show that the ergodicity of the geodesic flow on $\mathbb{H}^{2} / \Gamma$ with the hyperbolic measure is closely related to the quantitative theory of Diophantine approximation on $\Gamma$ (see [22] for the qualitative theory). The relation between the Diophantine inequality and geodesics of $\mathbb{H}^{2}$ has also been considered by A. Haas [5] and A. Haas and C. Series [6] to discuss the Lagrange spectrum of the approximation on $\Gamma$. The "height" of the $\Gamma$-congruent family of geodesics plays an important role in their discussion.

This idea is also applicable to the theory of Diophantine approximations for complex numbers, where we have to consider $\mathbb{H}^{3}$ (see [18]).

Since $\infty \in P$, there exists

$$
U_{\lambda}=\left(\begin{array}{cc}
1 & \lambda \\
0 & 1
\end{array}\right) \in \Gamma, \quad \lambda \in \mathbb{R}_{+}
$$

such that

$$
\left\{U_{\lambda}^{k}: k \in \mathbb{Z}\right\}=\Gamma_{\infty},
$$

where $\Gamma_{\infty}$ denotes the subgroup of $\Gamma$ that fixes $\infty$. We define the fundamental region $\mathcal{F}$ of $\Gamma$ by

$$
\mathcal{F}=\{z=x+i y:-\lambda / 2<x<\lambda / 2, y>0\} \cap \bigcap_{g \in \Gamma \backslash \Gamma_{\infty}}\{z:|c(g) z+d(g)|>1\} .
$$

Then $\mathcal{F}$ is a hyperbolic polygon and each side of it is an arc of the isometric circle of an element $g \in \Gamma$. The image of this side under $g$ is also a side of $\mathcal{F}$, which is an arc of the isometric circle of $g^{-1}$. We identify all such pairs and obtain a hyperbolic surface. It is well-known that the hyperbolic metric $d s=\sqrt{d x^{2}+d y^{2}} / y$ and the hyperbolic measure $d \mu=d x d y / y^{2}$ on $\mathbb{H}^{2}$ are invariant under the $\Gamma$-action over $\mathbb{H}^{2}$.

Theorem 1. Let

$$
t_{0}=\frac{1}{2} \min _{g \in \Gamma \backslash \Gamma_{\infty}}|c(g)| .
$$

Then

$$
\lim _{N \rightarrow \infty} \frac{\sharp\left\{g(\infty):|x-g(\infty)|<t / c^{2}(g),|c(g)| \leq N, g \in \Gamma\right\}}{\ln N}=\frac{4 \lambda t}{\pi \mu(\mathcal{F})}
$$

for any $t, 0<t<t_{0}$ (a.e. $\left.x \in \mathbb{R} \backslash P\right)$. 
The proof of this theorem is basically the same as that of the main result in [18] for the imaginary quadratic field case with the hyperbolic upper half-space. So we only give a sketch. We start with some lemmas.

We denote by $\gamma(x, y)$ the geodesic curve with initial point $x$ and terminal point $y$ for $(x, \beta) \in(\mathbb{R} \cup\{\infty\})^{2} \backslash\{$ diagonal $\}$. We also denote by

$$
F_{t}(g(\infty)), \quad t>0
$$

the circle which is tangent to the real line at $a(g) / c(g)$ with radius $t / c^{2}(g)$ for $g \notin \Gamma_{\infty}$ and $\{x+i y: y=1 / 2 t\}$ for $g \in \Gamma_{\infty}$.

We show the following:

Lemma 1. If $t>0$, then

$$
h\left(F_{t}(g(\infty))\right)=F_{t}(h g(\infty)) \quad \text { for any } h \text { and } g \in \Gamma .
$$

Proof. This follows from the fact that $F_{t}(g(\infty))$ is an image of $\{x+i y: y=1 / 2 t\}$, which forms an invariant family of circles under the $\Gamma$-action.

Lemma 2. For any $k>0$,

$$
|x-g(\infty)|<t / c^{2}(g)
$$

if and only if $\gamma(\infty, x)$ and $\bigcap F_{t}(g(\infty))$ do not cross each other.

If $t<t_{0}$, then we see that $\left\{F_{t}(g(\infty))\right\}$ is a disjoint family of circles, that is,

$$
F_{t}(g(\infty)) \cap F_{t}(h(\infty))=\emptyset \quad \text { if } g(\infty) \neq h(\infty) .
$$

Thus we have the following:

Lemma 3. If $0<t<t_{0}$, then every point of $F_{t}(g(\infty)) \backslash(\mathbb{R} \cup\{\infty\})$ is congruent to some point of $F_{t}(\infty) \cap(\mathcal{F} \backslash\{\infty\})$. In particular, if $p \in F_{t}(g(\infty)) \backslash(\mathbb{R} \cup\{\infty\})$, then there exists $h \in \Gamma$ such that $h(p)=x+i y,-\lambda / 2<x<\lambda / 2$ and $y=1 / 2 t$.

Proof of Theorem 1. Let $\mathbf{T}\left(\mathbb{H}^{2}\right)$ and $\mathbf{T}(\mathcal{F})$ be the unit tangent bundles of $\mathbb{H}^{2}$ and $\mathcal{F}$, respectively. We consider the geodesic flows $f_{s}$ and $\hat{f}_{s}$ on $\mathbf{T}\left(\mathbb{H}^{2}\right)$ and $\mathbf{T}(\mathcal{F})$, respectively. For $\omega^{*} \in \mathbf{T}\left(\mathbb{H}^{2}\right)$, there is a unique geodesic $(x, \beta)$ passing tangentially through $\omega^{*}$. If $x \neq \infty$ and $\beta \neq \infty$, then we denote by $s$ the (signed) hyperbolic length from the top of the geodesic arc $(x, \beta)$ to $\omega$, which is the base point of $\omega^{*}$. If $x=\infty$ (or $\left.\beta=\infty\right)$, then we denote by $s$ the hyperbolic length from the point $\beta+i$ or $x+i$ to $\omega$, respectively. Thus we can parameterize $\omega^{*} \in \mathbf{T}\left(\mathbb{H}^{2}\right)$ by $(x, \beta, s) \in\left((\mathbb{R} \cup\{\infty\})^{2} \backslash\{\right.$ diagonal $\left.\}\right) \times \mathbb{R}$. So if $0<t<t_{0}$, we see from Lemmas 2 and 3 that

$$
\begin{aligned}
\mid \sharp\{g(\infty) & \left.:|x-g(\infty)|<t / c^{2}(g),|c(g)| \leq N, g \in \Gamma\right\} \\
& -\sharp\left\{s: \begin{array}{l}
f_{s}\left(\infty, x,-\ln \left(4 t_{0}+1\right)\right) \text { crosses a circle } F_{t}(g(\infty)) \text { from } \\
\text { outside at time } s, 0<s \leq \ln \left(4 t_{0}+1\right)-\ln t+2 \ln N
\end{array}\right\} \mid \leq 1
\end{aligned}
$$


and

$$
\begin{aligned}
\sharp\left\{s: \begin{array}{l}
f_{s}\left(\infty, x,-\ln \left(4 t_{0}+1\right)\right) \text { crosses a circle } F_{t}(g(\infty)) \text { from } \\
\text { outside at time } s, 0<s \leq \ln \left(4 t_{0}+1\right)-\ln t+2 \ln N
\end{array}\right\} \\
=\sharp\left\{\begin{array}{l}
s: \begin{array}{l}
\hat{f}_{s}\left(\omega^{*}\right) \text { crosses } F_{t}(\infty) \text { from below at time } s, \\
0<s \leq \ln \left(4 t_{0}+1\right)-\ln t+2 \ln N
\end{array}
\end{array}\right\},
\end{aligned}
$$

where $\omega^{*} \in \mathbf{T}(\mathcal{F})$ is the point corresponding to $\left(\infty, x,-\ln \left(4 t_{0}+1\right)\right) \in \mathbf{T}\left(\mathbb{H}^{2}\right)$.

Now we apply the individual ergodic theorem for $(\mathbf{T}(\mathcal{F}), \hat{f}, \hat{\mu})$ to our problem. Here, the hyperbolic measure $\hat{\mu}$ on $\mathbf{T}(\mathcal{F})$ induced from $\mu$ is given by

$$
\hat{\mu}=\frac{d x d \beta d s}{(x-\beta)^{2}}
$$

if we parameterize a point in $\mathbf{T}(\mathcal{F})$ by $(x, \beta, s)$.

Proposition 1. If we fix $t, 0<t<t_{0}$, then

$$
\begin{aligned}
\lim _{u \rightarrow \infty} \frac{\sharp\left\{s: f_{s}\left(\omega^{*}\right) \text { crosses } F_{t}(\infty) \text { from below, } 0<s<u\right\}}{u} & \\
= & \frac{\mu\{x+i y \in \mathcal{F}: y>1 / 2 t\}}{\pi \mu(\mathcal{F})}
\end{aligned}
$$

for a.e. $\omega^{*} \in \mathbf{T}(\mathcal{F})$.

Moreover, by using an approximation method on $t$, we have

Proposition 2. For a.e. $\omega^{*} \in \mathbf{T}(\mathcal{F})$,

$$
\begin{aligned}
\lim _{u \rightarrow \infty} \frac{\sharp\left\{s: f_{s}\left(\omega^{*}\right) \text { crosses } F_{t}(\infty) \text { from below, } 0<s<u\right\}}{u} & \\
= & \frac{\mu\{x+i y \in \mathcal{F}: y>1 / 2 t\}}{\pi \mu(\mathcal{F})}
\end{aligned}
$$

for any $t, 0<t<t_{0}$.

Furthermore, it is possible to show that if $\omega^{*}=(x, \beta, s) \in \mathbf{T}(\mathcal{F})$ has the above property, then for any $x^{\prime} \in \mathbb{R} \cup\{\infty\}$ and $s^{\prime} \in \mathbb{R}, \omega^{* *}=\left(x^{\prime}, \beta, s^{\prime}\right)$ also has the same property. Since the hyperbolic length between $x+\left(4 t_{0}+1\right) i$ and $x+(1 / N) i$ is equal to $\ln N+\ln \left(4 t_{0}+1\right)$, we have

$$
\begin{aligned}
& \lim _{N \rightarrow \infty} \frac{\sharp\left\{g(\infty):|x-g(\infty)|<t / c^{2}(g),|c(g)| \leq N, g \in \Gamma\right\}}{\ln N} \\
&=2 \frac{\mu\{x+i y \in \mathcal{F}: y>1 / 2 t\}}{\pi \mu(\mathcal{F})}=\frac{4 \lambda t}{\pi \mu(\mathcal{F})}
\end{aligned}
$$

for any $t, 0<t<t_{0}$ and a.e. $x \in \mathbb{R} \backslash P$. 
We apply the theorem to the Hecke group of index $k, G_{k}, 3 \leq k \leq \infty$, and its congruent subgroups $G_{k}(m)$. Here $G_{k}$ is the group generated by

$$
\left(\begin{array}{cc}
0 & 1 \\
-1 & 0
\end{array}\right) \text { and }\left(\begin{array}{cc}
1 & \lambda_{k} \\
0 & 1
\end{array}\right)
$$

where $\lambda_{k}=2 \cos (\pi / k)$ for $k \geq 3$ (and $=2$ when $k=\infty$ ), and $G_{k}(m)$ the subgroup of $G_{k}$ defined by

$$
G_{k}(m)=\left\{g \in G_{k}: g \equiv\left(\begin{array}{cc} 
\pm 1 & 0 \\
0 & \pm 1
\end{array}\right) \bmod \left(m \cdot \lambda_{k}\right)\right\}
$$

where $\left(m \cdot \lambda_{k}\right)$ denotes the ideal generated by $m \cdot \lambda_{k}$ with a positive integer $m$.

A fundamental region $\mathcal{F}_{k}$ of $G_{k}$ is given by

$$
\mathcal{F}_{k}=\left\{x+i y:-\cos \pi n<x \leq \cos \pi k, x^{2}+y^{2}>1, y>0\right\} .
$$

Thus we see that $G_{k}$ is of the first kind and

$$
P=P_{k}=G_{k}(\infty)=\left\{g(\infty): g \in G_{k}\right\}
$$

if $k \neq \infty$. In this case, we have

$$
\lim _{N \rightarrow \infty} \frac{\sharp\left\{g(\infty):|x-g(\infty)|<t / c^{2}(g),|c(g)| \leq N, g \in G_{k}\right\}}{\ln N}=\frac{4 k \lambda_{k} t}{(k-2) \pi^{2}}
$$

for any $t, 0<t<1 / 2$, and a.e. $x \in \mathbb{R} \backslash P_{k}$. We can also apply Theorem 1 to $G_{k}(m)$. Then, in this case, the set of parabolic points of $G_{k}$ is divided into a finite number of disjoint sets. We put

$$
t_{m}=\frac{1}{2} \min _{g \in G_{k}(m) \backslash G_{k}(m)_{\infty}}|c(g)| .
$$

There exists a constant $C>0$ such that

$$
\lim _{N \rightarrow \infty} \frac{\sharp\left\{g(\infty):|x-g(\infty)|<t / c^{2}(g),|c(g)| \leq N, g \in G_{k}(m)\right\}}{\ln N}=C t
$$

for any $t, 0<t<t_{m}$, and a.e. $x \in \mathbb{R}$. Since $G_{k}(m)$ is a subgroup of $G_{k}$, for each cusp of the fundamental region of $G_{k}(m)$ there exists $g_{\eta} \in G_{k}$ such that $g_{\eta}(\eta)=\infty$. It is obvious that $g_{\eta} \mathcal{F}$ is a fundamental region of $g_{\eta} G_{k}(m) g_{\eta}^{-1}$. Since $G_{k}(m)$ is normal, $g_{\eta} \mathcal{F}$ is a fundamental region of $G_{k}(m)$. This means the "width" of the cusp $\eta$ is the same as that of $\infty$. Thus we have

$$
\lim _{N \rightarrow \infty} \frac{\sharp\left\{g(\infty):|x-g(\infty)|<t / c^{2}(g),|c(g)| \leq N, g \in G_{k}, \exists \hat{g} \in G_{k}(m), g(\infty)=\hat{g}(\eta)\right\}}{\ln N}=C t
$$

for any $t, 0<t<t_{m}$, and a.e. $x \in \mathbb{R}$. Moreover, it turns out that $t_{m} \rightarrow \infty$ as $m \rightarrow \infty$. This shows the following: 
Corollary. For a.e. $x \in \mathbb{R}$,

$$
\lim _{N \rightarrow \infty} \frac{\sharp\left\{g(\infty):|x-g(\infty)|<t / c^{2}(g),|c(g)| \leq N, g \in G_{k}\right\}}{\ln N}=\frac{4 k \lambda_{k} t}{(k-2) \pi^{2}}
$$

for any $t>0$.

Remark. The above proof (of this corollary) shows the equidistribution property (a.e.) of solutions associated to cusps. We refer to R. Moeckel [15] for the original idea of this method.

\section{Rosen continued fractions}

Given any element of $G_{k}$ of the form

$$
\left(\begin{array}{ll}
p & \cdot \\
q & \cdot
\end{array}\right)
$$

we have $g(\infty)=p / q$; moreover, for any $\tilde{g} \in G_{k}$ with $\tilde{g}(\infty)=p / q$ we have

$$
\tilde{g}=\left(\begin{array}{ll}
p & \cdot \\
q & \cdot
\end{array}\right) \text { or } \quad\left(\begin{array}{ll}
-p & \cdot \\
-q & \cdot
\end{array}\right)
$$

So, for any parabolic point of $G_{k}, p$ and $q>0$ are uniquely determined.

We define the $\lambda_{k}$-nearest continued fraction transformation of $\left[-\lambda_{k} / 2, \lambda_{k} / 2\right)$ onto itself by

$$
S(x))= \begin{cases}\left|\frac{1}{x}\right|-\left[\left|\frac{1}{x}\right|\right]_{k} & \text { for } x \neq 0, \\ 0 & \text { for } x=0\end{cases}
$$

where $[w]_{k}=b \lambda_{k}, b \in \mathbb{Z}$, when $w \in\left[b-\lambda_{k} / 2, b+\lambda_{k} / 2\right)$. We put

$$
\varepsilon_{n}=\varepsilon_{n}(x)=\operatorname{sgn}\left(S^{n-1}(x)\right) \quad \text { and } \quad a_{n}=a_{n}(x)=\left[\left|\frac{1}{S^{n-1}(x)}\right|\right]_{k}
$$

for any $n \geq 1$ and have a continued fraction expansion

$$
x=\frac{\varepsilon_{1} \mid}{\mid a_{1}}+\frac{\varepsilon_{2} \mid}{\mid a_{2}}+\cdots .
$$

We call this expansion the Rosen continued fraction expansion of $x$. In the case of $k=3$, it is the classical nearest integer continued fraction expansion. In general, if a continued fraction, either finite or infinite, is given by some $x$ as its Rosen continued fraction expansion, we call it a Rosen continued fraction. We define the principal convergent $p_{n} / q_{n}, n \geq 0$, by

$$
\left(\begin{array}{ll}
p_{-1} & p_{0} \\
q_{-1} & q_{0}
\end{array}\right)=\left(\begin{array}{ll}
1 & 0 \\
0 & 1
\end{array}\right)
$$


and

$$
\left(\begin{array}{ll}
p_{n-1} & p_{n} \\
q_{n-1} & q_{n}
\end{array}\right)=\left(\begin{array}{ll}
0 & \varepsilon_{1} \\
1 & a_{1}
\end{array}\right)\left(\begin{array}{ll}
0 & \varepsilon_{2} \\
1 & a_{2}
\end{array}\right) \cdots\left(\begin{array}{ll}
0 & \varepsilon_{n} \\
1 & a_{n}
\end{array}\right) \quad \text { for } n \geq 1 .
$$

It is easy to see that $q_{n}>0$ for any $n \geq 0$.

Lemma 4. We have

$$
\frac{1}{q_{n}\left(q_{n+1}+q_{n}\right)} \leq\left|x-\frac{p_{n}}{q_{n}}\right| \leq \begin{cases}\frac{1}{q_{n}^{2}\left(1-\lambda_{k} / 2\right)} & \text { if } k \text { is even, } \\ \frac{1}{q_{n}^{2}\left(1 / R_{k}-\lambda_{k} / 2\right)} & \text { if } k \text { is odd },\end{cases}
$$

where $R_{k}$ is the positive root of $X^{2}+(2-\lambda) X-1=0$.

Proof. We have

$$
T^{n}(x)=\left(\begin{array}{ll}
p_{n-1} & p_{n} \\
q_{n-1} & q_{n}
\end{array}\right)^{-1}(x),
$$

and therefore (see also [7]),

$$
x=\left(\begin{array}{ll}
p_{n-1} & p_{n} \\
q_{n-1} & q_{n}
\end{array}\right)\left(T^{n}(x)\right)=\frac{p_{n-1} T^{n} x+p_{n}}{q_{n-1} T^{n} x+q_{n}} .
$$

Then we have (see e.g. [7, p. 27]),

$$
\left|x-\frac{p_{n}}{q_{n}}\right|=\frac{1}{q_{n}^{2}} \frac{1}{q_{n+1} / q_{n}+T^{n+1} x} .
$$

Since $q_{n+1} / q_{n}>1$ if $k$ is even (and $q_{n+1} / q_{n}>1 / R_{k}$ if $k$ is odd), the result follows.

Lemma 5. For a.e. $x \in \mathbb{I}$,

$$
\lim _{n \rightarrow \infty} \frac{1}{n} \ln q_{n}
$$

exists and is equal to half the entropy $h_{k}$ of the Rosen map with respect to the absolutely continuous invariant probability measure.

Proof. Let $\mu$ be the absolutely continuous invariant probability measure for $T$. Since $(T, \mu)$ is ergodic (see [3]), the Shannon-McMillan-Breiman theorem implies that the entropy $h_{k}$ of the Rosen map is given by

$$
h_{k}=\lim _{n \rightarrow \infty} \frac{1}{n} \ln \mu\left(\Delta\left[\varepsilon_{1}: r_{1}, \ldots, \varepsilon_{n}: r_{n}\right]\right) \quad \text { a.e. }
$$

We can replace $\mu$ by the normalized Lebesgue measure $m$ because $\mu$ has a positive density function bounded away from 0 and bounded from above (see [3]), that is,

$$
h_{k}=\lim _{n \rightarrow \infty} \frac{1}{n} \ln m\left(\Delta\left[\varepsilon_{1}: r_{1}, \ldots, \varepsilon_{n}: r_{n}\right]\right) \text { a.e. }
$$


From Lemma 4, it turns out that

$$
\lim _{n \rightarrow \infty} \frac{1}{n} \ln m\left(\Delta\left[\varepsilon_{1}: r_{1}, \ldots, \varepsilon_{n}: r_{n}\right]\right)=2 \lim _{n \rightarrow \infty} \frac{1}{n} \ln q_{n}
$$

if the limit on the left hand side exists. Thus we have

$$
\lim _{n \rightarrow \infty} \frac{1}{n} \ln q_{n}=\frac{h_{k}}{2}
$$

for a.e. $x \in \mathbb{I}$.

Now we denote by $\mathcal{L} g_{k}$ the Legendre constant of Rosen continued fractions of index $k$, that is, the following hold:

(1) for $c \leq \mathcal{L} g_{k}$ and $x \in\left[-\lambda_{k} / 2, \lambda_{k} / 2\right)$, and $\left(\begin{array}{c}p \\ q\end{array}:\right) \in G_{k}, q \neq 0$, if $|x-p / q|<c / q^{2}$, then $p / q=p_{n} / q_{n}$ for some $n \geq 0$,

(2) for $c>\mathcal{L} g_{k}$, there exist $x \in\left[-\lambda_{k} / 2, \lambda_{k} / 2\right)$ and $\left(\begin{array}{c}p \\ q\end{array}:\right) \in G_{k}, q \neq 0$, such that $|x-p / q|<c / q^{2}$ and $p / q \neq p_{n} / q_{n}$ for any $n \geq 0$.

As mentioned in the introduction, the existence of $\mathcal{L} g_{k}$ was shown in [21].

On the other hand, we denote by $\mathcal{L} e_{k}$ the Lenstra constant of Rosen continued fractions of index $k$. This means that for almost every $x \in\left[-\lambda_{k} / 2, \lambda_{k} / 2\right)$,

$$
\lim _{N \rightarrow \infty} \frac{1}{N} \sharp\left\{n: 1 \leq n \leq N, \Theta_{n}(x)<t\right\} \begin{cases}=C_{k} t & \text { for any } 0<t \leq \mathcal{L} e_{k}, \\ <C_{k} t & \text { for any } t>\mathcal{L} e_{k},\end{cases}
$$

where $C_{k}$ is an absolute constant, which is given in [3], and

$$
\Theta_{n}=q_{n}^{2}\left|x-\frac{p_{n}}{q_{n}}\right|
$$

We will prove the following:

Theorem 2. For any $k \geq 3$, we have $\mathcal{L} g_{k}=\mathcal{L} e_{k}$.

Remark. As a direct consequence of Theorem 2 and Corollary 4.1 of [3], we obtain the Legendre constant of Rosen continued fractions as follows:

$$
\mathcal{L} g_{k}=\mathcal{L} e_{k}= \begin{cases}\frac{\lambda_{k}}{\lambda_{k}+2} & \text { if } k \geq 4 \text { is an even integer, } \\ \frac{R_{k}}{R_{k}+1} & \text { if } k \geq 3 \text { is an odd integer. }\end{cases}
$$

The proof of Theorem 2 is based upon the following two propositions.

Proposition 3. For any $k \geq 3$, we have $\mathcal{L} g_{k} \leq \mathcal{L} e_{k}$. 
Proof. Suppose $0 \leq t \leq \mathcal{L} g_{k}$. From Corollary of $\S 2$, we have, for a.e. $x \in\left[-\lambda_{k} / 2, \lambda_{k} / 2\right)$,

$$
\begin{aligned}
& \lim _{N \rightarrow \infty} \frac{\sharp\left\{g(\infty):|x-g(\infty)|<t / c^{2}(g),|c(g)|=q_{n}, g \in G_{k}, \text { for some } 0 \leq n \leq N\right\}}{\ln q_{N}} \\
& \quad=\lim _{N \rightarrow \infty} \frac{\sharp\left\{g(\infty):|x-g(\infty)|<t / c^{2}(g),|c(g)| \leq q_{N}, g \in G_{k}\right\}}{\ln q_{N}}=\frac{4 k \lambda_{k} t}{(k-2) \pi^{2}} .
\end{aligned}
$$

We note that

$$
\begin{aligned}
& \lim _{N \rightarrow \infty} \frac{\sharp\left\{g(\infty):|x-g(\infty)|<t / c^{2}(g),|c(g)|=q_{n}, g \in G_{k}, \text { for some } 0 \leq n \leq N\right\}}{\ln q_{N}} \\
& =\lim _{N \rightarrow \infty} \frac{\frac{1}{N} \sharp\left\{g(\infty):|x-g(\infty)|<t / c^{2}(g),|c(g)|=q_{n}, g \in G_{k}, \text { for some } 0 \leq n \leq N\right\}}{\frac{1}{N} \ln q_{N}} .
\end{aligned}
$$

From Lemma 5, the denominator of the right hand side converges to $h_{k} / 2$ (a.e.), and we see that the numerator converges (a.e.) to

$$
\frac{4 k \lambda_{k} t h_{k}}{2(k-2) \pi^{2}}
$$

This means

$$
\lim _{N \rightarrow \infty} \frac{1}{N} \sharp\{n: 1 \leq n \leq N, \Theta<t\}=\frac{4 k \lambda_{k} t h_{k}}{2(k-2) \pi^{2}} \quad \text { a.e. }
$$

for $0 \leq t \leq \mathcal{L} g_{k}$.

Proposition 4. For any $k \geq 3$, we have $\mathcal{L} g_{k} \geq \mathcal{L} e_{k}$.

Proof. Suppose that $t>\mathcal{L} g_{k}$. Then there exist $x \in\left[-\lambda_{k} / 2, \lambda_{k} / 2\right)$ and $(\underset{q}{p}:) \in G_{k}$ such that

$$
\left|x-\frac{p}{q}\right|<\frac{t}{q^{2}} \quad \text { and } \quad \frac{p}{q} \neq \frac{p_{n}}{q_{n}} \quad \text { for any } n \geq 0 .
$$

From this inequality, there exists $\varepsilon>0$ such that

$$
\left|x-\frac{p}{q}\right|<\frac{t}{q^{2}}-\varepsilon
$$

If $y \in\left[-\lambda_{k} / 2, \lambda_{k} / 2\right)$ is sufficiently close to $x$, i.e.

$$
|x-y|<\frac{\varepsilon}{2},
$$

then

$$
\left|y-\frac{p}{q}\right|<\frac{t}{q^{2}}-\frac{\varepsilon}{2} .
$$

Moreover, there exists a positive integer $M_{0}$ such that the equality

$$
\left(\varepsilon_{i}(x), a_{i}(x)\right)=\left(\varepsilon_{i}(y), a_{i}(y)\right) \quad \text { for } 1 \leq i \leq M_{0}
$$

implies that (2) holds. 
Now we look at the expansions of $p / q$ and $x$. Since $p / q$ is a parabolic point of $G_{k}$, it is easy to see that $p / q$ has a finite Rosen expansion, say,

$$
\frac{p}{q}=\frac{\hat{\varepsilon}_{1} \mid}{\mid \hat{a}_{1}}+\cdots+\frac{\hat{\varepsilon}_{n} \mid}{\mid \hat{a}_{n}} .
$$

This means there exists $m, 1 \leq m \leq n$, such that

$$
\left(\varepsilon_{i}, a_{i}\right)=\left(\hat{\varepsilon}_{1}, \hat{a}_{i}\right) \quad \text { for } 1 \leq i \leq m-1 \quad \text { and } \quad\left(\varepsilon_{m}, a_{m}\right) \neq\left(\hat{\varepsilon}_{m}, \hat{a}_{m}\right)
$$

with

$$
x=\frac{\varepsilon_{1} \mid}{\mid a_{1}}+\cdots+\frac{\varepsilon_{m} \mid}{\mid a_{m}}+\frac{\varepsilon_{m+1} \mid}{\mid a_{m+1}}+\cdots+\frac{\varepsilon_{n} \mid}{\mid a_{n}}+\cdots .
$$

Here we may assume that $M_{0}>n$. We choose a positive integer $M_{1}$ sufficiently large and define

$$
\hat{a}_{0}=M_{1} \lambda_{k}
$$

We see that

$$
\frac{\hat{\varepsilon}_{0} \mid}{\mid \hat{a}_{0}}+\frac{\hat{\varepsilon}_{1} \mid}{\mid \hat{a}_{1}}+\cdots+\frac{\hat{\varepsilon}_{n} \mid}{\mid \hat{a}_{n}}
$$

is a Rosen continued fraction, where $\hat{\varepsilon}_{0}=+1$. We fix any finite Rosen continued fraction

$$
\frac{\varepsilon_{1}^{\prime} \mid}{\mid b_{1}}+\cdots+\frac{\varepsilon_{l}^{\prime} \mid}{\mid b_{l}}
$$

so that

$$
\frac{\varepsilon_{1}^{\prime} \mid}{\mid b_{1}}+\cdots+\frac{\varepsilon_{l}^{\prime} \mid}{\mid b_{l}}+\frac{\hat{\varepsilon}_{0} \mid}{\mid \hat{a}_{0}}+\frac{\hat{\varepsilon}_{1} \mid}{\mid \hat{a}_{1}}+\cdots+\frac{\hat{\varepsilon}_{n} \mid}{\mid \hat{a}_{n}}
$$

and

$$
\frac{\varepsilon_{1}^{\prime} \mid}{\mid b_{1}}+\cdots+\frac{\varepsilon_{l}^{\prime} \mid}{\mid b_{l}}+\frac{\hat{\varepsilon}_{0} \mid}{\mid \hat{a}_{0}}+\frac{\varepsilon_{1} \mid}{\mid a_{1}}+\cdots+\frac{\varepsilon_{m} \mid}{\mid a_{m}}+\frac{\varepsilon_{m+1} \mid}{\mid a_{m+1}}+\cdots+\frac{\varepsilon_{n} \mid}{\mid a_{n}}+\cdots+\frac{\varepsilon_{M_{0}} \mid}{\mid a_{M_{0}}}+\cdots
$$

are also Rosen continued fractions. Suppose that $y_{0} \in\left[-\lambda_{k} / 2, \lambda_{k} / 2\right)$ with the Rosen expansion of the form

$\frac{\varepsilon_{1}^{\prime} \mid}{\mid b_{1}}+\cdots+\frac{\varepsilon_{l}^{\prime} \mid}{\mid b_{l}}+\frac{\hat{\varepsilon}_{0} \mid}{\mid \hat{a}_{0}}+\frac{\varepsilon_{1} \mid}{\mid a_{1}}+\cdots+\frac{\varepsilon_{m} \mid}{\mid a_{m}}+\frac{\varepsilon_{m+1} \mid}{\mid a_{m+1}}+\cdots+\frac{\varepsilon_{n} \mid}{\mid a_{n}}+\cdots+\frac{\varepsilon_{M_{0}} \mid}{\mid a_{M_{0}}}+$ (free)

and

$$
\frac{P}{Q}=\frac{\varepsilon_{1}^{\prime} \mid}{\mid b_{1}}+\cdots+\frac{\varepsilon_{l}^{\prime} \mid}{\mid b_{l}}+\frac{\hat{\varepsilon}_{0} \mid}{\mid \hat{a}_{0}}+\frac{\hat{\varepsilon}_{1} \mid}{\mid \hat{a}_{1}}+\cdots+\frac{\hat{\varepsilon}_{n-1} \mid}{\mid \hat{a}_{n-1}}+\frac{\hat{\varepsilon}_{n} \mid}{\mid \hat{a}_{n}} .
$$

We note that $P$ and $Q$ are uniquely determined by $(\stackrel{P}{Q}:) \in G_{k}$ and $Q>0$. We also note that $y:=S^{l+1}\left(y_{0}\right)$ has Rosen continued fraction

$$
\frac{\varepsilon_{1} \mid}{\mid a_{1}}+\cdots+\frac{\varepsilon_{m} \mid}{\mid a_{m}}+\frac{\varepsilon_{m+1} \mid}{\mid a_{m+1}}+\cdots+\frac{\varepsilon_{n} \mid}{\mid a_{n}}+\cdots+\frac{\varepsilon_{M_{0}} \mid}{\mid a_{M_{0}}}+\text { (free) }
$$


Lenstra constant associated to Rosen continued fractions

67

which satisfies (2). We put

$$
\left(\begin{array}{cc}
P_{n+l} & P_{n+l+1} \\
Q_{n+l} & Q_{n+l+1}
\end{array}\right)=\left(\begin{array}{cc}
0 & \varepsilon_{1}^{\prime} \\
1 & b_{1}
\end{array}\right) \cdots\left(\begin{array}{cc}
0 & \varepsilon_{l}^{\prime} \\
1 & b_{l}
\end{array}\right)\left(\begin{array}{ll}
0 & \hat{\varepsilon}_{0} \\
1 & \hat{a}_{0}
\end{array}\right)\left(\begin{array}{ll}
0 & \hat{\varepsilon}_{1} \\
1 & \hat{a}_{1}
\end{array}\right) \cdots\left(\begin{array}{ll}
0 & \hat{\varepsilon}_{n} \\
1 & \hat{a}_{n}
\end{array}\right),
$$

which implies $(P, Q)=\left(P_{n+l+1}, Q_{n+l+1}\right)$, and we will estimate

$$
\left|y_{0}-\frac{P_{n+l+1}}{Q_{n+l+1}}\right| \text {. }
$$

We also define

$$
\begin{aligned}
\left(\begin{array}{ll}
P_{l-1} & P_{l} \\
Q_{l-1} & Q_{1}
\end{array}\right) & =\left(\begin{array}{ll}
0 & \varepsilon_{1}^{\prime} \\
1 & b_{1}
\end{array}\right) \cdots\left(\begin{array}{ll}
0 & \varepsilon_{l}^{\prime} \\
1 & b_{l}
\end{array}\right), \\
\left(\begin{array}{ll}
P_{l} & P_{l+1} \\
Q_{l} & Q_{1+1}
\end{array}\right) & =\left(\begin{array}{ll}
0 & \varepsilon_{1}^{\prime} \\
1 & b_{1}
\end{array}\right) \cdots\left(\begin{array}{ll}
0 & \varepsilon_{l}^{\prime} \\
1 & b_{l}
\end{array}\right)\left(\begin{array}{ll}
0 & \hat{\varepsilon}_{0} \\
1 & \hat{a}_{0}
\end{array}\right) .
\end{aligned}
$$

We denote by $U$ the linear fractional transformation defined by $\left(\begin{array}{cc}P_{l} & P_{l+1} \\ Q_{l} & Q_{l+1}\end{array}\right)$. Then it is easy to see that

$$
U\left(\frac{p}{q}\right)=\frac{P}{Q} \quad \text { and } \quad U(y)=y_{0}
$$

Thus

$$
\left|y_{0}-\frac{P_{n+l+1}}{Q_{n+l+1}}\right|=\left|U\left(\frac{p}{q}\right)-U(y)\right|
$$

and the following holds:

$$
\begin{aligned}
\left|U\left(\frac{p}{q}\right)-U(y)\right|= & \left|\frac{P_{l} y+P_{l+1}}{Q_{l} y+Q_{l+1}}-\frac{P_{l} \frac{p}{q}+P_{l+1}}{Q_{l} \frac{p}{q}+Q_{l+1}}\right| \\
= & \left|\left(\frac{P_{l} y+P_{l+1}}{Q_{l} y+Q_{l+1}}-\frac{P_{l+1}}{Q_{l+1}}\right)+\left(\frac{P_{l+1}}{Q_{l+1}}-\frac{P_{l} \frac{p}{q}+P_{l+1}}{Q_{l} \frac{p}{q}+Q_{l+1}}\right)\right| \\
= & \mid \frac{\frac{p}{q}}{Q_{l+1}\left(Q_{l} y+Q_{l+1}\right)}+\frac{\frac{p}{Q_{l+1}\left(Q_{l} \frac{p}{q}+Q_{l+1}\right)} \mid}{\leq} \\
& +\left|\frac{\frac{p}{q}}{Q_{l+1}\left(Q_{l} y+Q_{l+1}\right) \mid}\right| \\
\leq & \frac{\frac{p}{q}}{\left|Q_{l+1}\left(Q_{l} y+Q_{l+1}\right)\right|}\left(\frac{t}{q}+\frac{1}{2}\right) \\
& +\left|\frac{p}{q}\right|\left|\frac{1}{Q_{l+1}\left(Q_{l} \frac{p}{q}+Q_{l+1}\right)}-\frac{1}{Q_{l+1}\left(Q_{l} y+Q_{l+1}\right)}\right|
\end{aligned}
$$


From the definition of $Q_{n+l+1}$, we see

$$
q_{l+n+1}=p Q_{l}+q Q_{l+1} .
$$

Since $|y|<1,|p / q|$ cannot be large, $Q_{l+1}=\hat{a}_{0} Q_{l}+\hat{\varepsilon}_{0} Q_{l-1}$, and $Q_{l-1} / Q_{l}$ is bounded (see [3]), we see that

$$
\left|\frac{Q_{l} y}{Q_{l+1}}\right| \text { and }\left|\frac{Q_{l} \frac{p}{q}}{Q_{l+1}}\right|
$$

can be arbitrarily small and

$$
\frac{q Q_{l+1}}{Q_{l+n+1}}
$$

can be sufficiently close to 1 when we choose $M_{1}$ sufficiently large (note that $\hat{a}_{0}=M_{1} \lambda_{k}$ ).

In the above discussion, the choice of $M_{1}$ can be independent of $\left(\varepsilon_{1}^{\prime}, b_{1}\right), \ldots\left(\varepsilon_{l}^{\prime}, b_{l}\right)$. Thus we get

$$
\left|y_{0}-\frac{P}{Q}\right|<\frac{t}{Q^{2}} .
$$

It is obvious from the construction that

$$
\frac{P}{Q} \neq \frac{p_{u}}{q_{u}}
$$

for any $u \geq 0$. Now we pick up a "generic point" $w \in\left[-\lambda_{k} / 2, \lambda_{k} / 2\right)$. Then the ergodicity of $S$ with respect to $\mu_{k}$ yields

$$
\begin{gathered}
\lim _{N \rightarrow \infty} \frac{1}{N} \sharp\left\{1 \leq u \leq N:\left(\varepsilon_{u}(w), a_{u}(w)\right)=\left(\hat{\varepsilon}_{0}, \hat{a}_{0}\right),\left(\varepsilon_{u+1}(w), a_{u+1}(w)\right)=\left(\varepsilon_{1}, a_{1}\right), \ldots,\right. \\
\left.\left(\varepsilon_{u+n}(w), a_{u+n}(w)\right)=\left(\varepsilon_{n}, a_{n}\right), \ldots,\left(\varepsilon_{u+M_{0}}(w), a_{u+M_{0}}(w)\right)=\left(\varepsilon_{M_{0}}, a_{M_{0}}\right)\right\}
\end{gathered}
$$

$>0$.

Finally, we look at

$$
\begin{aligned}
& \frac{1}{\ln Q} \sharp\left\{1 \leq q \leq Q:\left|w-\frac{p}{q}\right|<\frac{t}{q^{2}}\right\} \\
&= \frac{1}{\ln Q} \sharp\left\{1 \leq q \leq Q:\left|w-\frac{p}{q}\right|<\frac{t}{q^{2}}, \frac{p}{q}=\frac{p_{n}}{q_{n}} \text { for some } n \geq 1\right\} \\
&+\frac{1}{\ln Q} \sharp\left\{1 \leq q \leq Q:\left|w-\frac{p}{q}\right|<\frac{t}{q^{2}}, \frac{p}{q} \neq \frac{p_{n}}{q_{n}} \text { for some } n \geq 1\right\} .
\end{aligned}
$$

From the above discussion, the second term has a positive "liminf" and then the first term cannot converge to $4 k \lambda_{k} t /(k-2) \pi^{2}$. Since this calculation holds for a.e. $w, t$ is larger than $\mathcal{L} e_{k}$. 
Consequently, we have shown the assertion of Theorem 2. The method of the proof in the above yields the following generalization:

Claim. Suppose that $T$ is a map of an interval onto itself that induces continued fraction expansions for real numbers in the domain interval. Moreover, assume

(i) Thas an absolutely invariant probability measure.

(ii) There exists a real number $M>0$ such that for any possible coefficient value $c$ larger than $M($ or $|c|>M)$, one can concatenate any admissible sequence after $c$ as an admissible sequence of continued fractions arising from $T$.

(iii) The Legendre constant of $T$ exists.

(iv) $t_{0}$ in Theorem 1 is larger than the Legendre constant.

Then the Lenstra constant exists and the Legendre and the Lenstra constants are equal.

From (1) in the proof of Proposition 3 together with Corollary 4.1 of [3], we have the explicit value of the entropy of the Rosen map.

Corollary. The entropy of the Rosen map with respect to the absolutely continuous invariant probability measure is equal to

$$
\frac{C(k-2) \pi^{2}}{2 k}
$$

with

$$
C= \begin{cases}\frac{1}{\ln \left\{\left(1+\cos \frac{\pi}{k}\right) / \sin \frac{\pi}{k}\right\}} & \text { if } k \text { is even, } \\ \frac{1}{\ln \left(1+R_{k}\right)} & \text { if } k \text { is odd. }\end{cases}
$$

Acknowledgments. The author is grateful to Thomas A. Schmidt for his careful reading of the first draft of this paper, helpful advice, and useful comments. The author also thanks the referee for suggestions to improve the presentation of the paper.

This research was supported by Bezoekersbeurs B 61-620 of the Nederlandse Organisatie voor Wetenschappelijk Onderzoek (NWO) and Grant-in Aid for Scientific Research 18340032 of Japan Society for the Promotion of Science.

\section{References}

[1] Ahlfors, L. V.: Möbius transformations in several dimensions. Minnesota Lecture Notes (1981) Zbl 0517.30001 MR 0725161

[2] Bosma, W., Jager, H., Wiedijk, F.: Some metrical observations on the approximation by continued fractions. Indag. Math. 45, 281-299 (1983) Zbl 0519.10043 MR 0718069

[3] Burton, R., Kraaikamp, C., Schmidt, T.: Natural extensions for the Rosen fractions. Trans. Amer. Math. Soc. 352, 1277-1298 (1999) Zbl 0938.11036 MR 1650073

[4] Doeblin, W.: Remarques sur la théorie métrique des fractions continues. Compos. Math. 7, 353-371 (1940) Zbl 0022.37001 MR 0002732

[5] Haas, A.: Diophantine approximation on hyperbolic Riemann surfaces. Acta Math. 156, 33 82 (1986) Zbl 0593.10028 MR 0822330 
[6] Haas, A., Series, C.: The Hurwitz constant and Diophantine approximation on Hecke groups. J. London Math. Soc. 34, 219-234 (1986) Zbl 0605.10018 MR 0856507

[7] Iosifescu, M., Kraaikamp, C.: Metrical Theory of Continued Fractions. Math. Appl. 547, Kluwer, Dordrecht (2002) Zbl 1122.11047 MR 1960327

[8] Ito, S.: Algorithms with mediant convergents and their metrical theory. Osaka J. Math. 26, 557-578 (1989) Zbl 0702.11046 MR 1021431

[9] Kraaikamp, C.: A new class of continued fraction expansions. Acta Arith. 57, 1-39 (1991) Zbl 0721.11029 MR 1093246

[10] Kraaikamp, C., Nakada, H., Schmidt, T.: Metric and arithmetic properties of mediant-Rosen maps. Acta Arith. 137, 235-234 (2009) Zbl pre05552262 MR 2506585

[11] Kraaikamp, C., Smeets, I., Schmidt, T.: Tong's spectrum for Rosen continued fractions. J. Théor. Nombres Bordeaux 19, 641-661 (2007) Zbl pre05302618 MR 2388792

[12] Lehner, J.: Discontinuous Groups and Automorphic Functions. Math. Surveys 8, Amer. Math. Soc. (1964) Zbl 0178.42902 MR 0164033

[13] Lehner, J.: Diophantine approximation on Hecke groups. Glasgow Math. J. 27, 117-127 (1985) Zbl 0576.10023 MR 0819833

[14] Lehner, J.: The local Hurwitz constant and Diophantine approximation on Hecke groups. Math. Comp. 55, 765-781 (1990) Zbl 0761.11020 MR 1035937

[15] Moeckel, R.: Geodesics on modular surfaces and continued fractions. Ergodic Theory Dynam. Systems 2, 69-83 (1982) Zbl 0497.10007 MR 0684245

[16] Nakada, H.: Metrical theory for a class of continued fraction transformations and their natural extensions. Tokyo J. Math. 4, 399-426 (1981) Zbl 0479.10029 MR 0646050

[17] Nakada, H.: Continued fractions, geodesic flows and Ford circles. In: Algorithms, Fractals and Dynamics, Y. Takahashi (ed.), Plenum, 179-191 (1995) Zbl 0868.30005 MR 0646050

[18] Nakada, H.: On metrical theory of Diophantine approximation over imaginary quadratic field. Acta Arith. 51, 399-403 (1988) Zbl 0666.10021 MR 0971089

[19] Natsui, R.: On the Legendre constant of $\alpha$-continued fractions. Preprint.

[20] J. Patterson, S.: Diophantine approximation in Fuchsian groups. Philos. Trans. Roy. Soc. London 282, 527-563 (1976) Zbl 0338.10028 MR 0568140

[21] Rosen, D., Schmidt, T.: Hecke groups and continued fractions. Bull. Austral. Math. Soc. 46, 459-474 (1992) Zbl 0754.11012 MR 1190349

[22] Sullivan, D.: Disjoint spheres, approximations by imaginary quadratic numbers, and the logarithm law for geodesics. Acta Math. 149, 215-239 (1983) Zbl 0517.58028 MR 0688349 